\title{
ANÁLISE FILOGENETICA DAS ESPÉCIES DE VESPAS SOCIAIS NEOTROPICAIS LEIPOMELES MOEBIUS, 1856 (HYMENOPTERA, VESPIDAE, EPIPONINI)
}

\author{
Gabriel Santos Vieira ${ }^{1}$; Sergio Ricardo Andena ${ }^{2}$ \\ 1. Bolsista PIBIC/CNPq, Graduando em Bacharelado em Ciências Biológicas, Universidade Estadual de Feira de Santana, \\ email: gabriels.vieira22@gmail.com \\ 2. Orientador, Departamento de Ciências Biológicas, Universidade Estadual de Feira de Santana, e-mail: \\ sergioricardoandena@gmail.com
}

PALAVRAS-CHAVE: Leipomeles; Filogenia; Monofilia.

\section{INTRODUÇÃO}

Leipomeles (Vespidae, Epiponini) foi descrito por Moebius (1856), como um gênero monotípico, o qual designou Leipomeles lamellaria [= Polistes dorsata Fabricius, 1804] como espécie tipo. Richards, 1978 reconhece duas espécies, L. dorsata e L. nana. Richards (1978) também descreve o gênero Marimbonda para duas espécies, M. albogrisea como espécie tipo, e M. pusilla (Ducke, 1904). Estes gêneros possuem como característica uma cerda curvada no terceiro segmento do palpo labial, característica essa compartilhada por Pseudopolybia, Parachartergus, Chartergellus e Nectarinella (Carpenter, 1991). Análises cladísticas realizadas por Carpenter (1991) e Carpenter e Wenzel (1994) posicionaram Leipomeles e Marimbonda como grupo irmão. Carpenter (2004) sinonimiza Leipomeles com Marimbonda baseado em características morfológicas e principalmente de arquitetura de ninho, fazendo com que o gênero possua quatro espécies válidas. O presente trabalho propõe a análise cladística das quatro espécies de Leipomeles, bem como testar o monofiletismo do gênero.

\section{METODOLOGIA}

Os espécimes estudados foram obtidos através de empréstimo do American Museum of Natural History (New York, USA), Museu Paraense Emilio Goeldi (Pará, Brasil), Instituto Nacional de Pesquisas da Amazônia (INPA, Manaus, Brasil), Museu de Zoologia da Universidade de São Paulo (São Paulo, Brasil), University of California Washington Center (UCDC), além da coleção entomológica Johan Becker do Museu de Zoologia da Universidade de Feira de Santana (MZFS). Os caracteres obtidos na análise morfológica foram somados aos já existentes na literatura, combinados e analisados segundo a metodologia desenvolvida por Hennig (1966) e sintetizada em Amorim (1997). Os caracteres foram codificados como binários ou estados múltiplos. A polaridade dos caracteres foi determinada através dos métodos propostos por Nixon \& Carpenter (1993), no qual os táxons terminais do grupo "interno" são tratados conjuntamente com os táxons terminais dos grupos "externos" na análise de parcimônia. Para a análise utilizamos o programa de parcimônia TNT usando como interface o programa WinClada versão 1.0. O algoritmo utilizado foi o de enumeração implícita, onde todas as possíveis árvores são testadas.

\section{RESULTADOS E DISCUSSÃO}

Foram levantados 39 caracteres, representados na lista abaixo, codificados como binários ou estados múltiplos em uma matriz (Tabela 1).

0. Terceiro Palpomero Labial: 0 - sem cerda curvada; 1 - com cerda

1. Número de segmentos do Palpo Maxilar: $0-6 ; 1-5$

2. Número de segmentos do Palpo Labial: $0-4 ; 1-3$

3. Cerdas no olho: 0 - presente; 1 - ausente

4. Clípeo: 0 - igual ou mais largo do que longo; 1 - mais longo do que largo

5. Clípeo em perfil: 0 - côncavo; 1 - achatado 
6. Ápice do clípeo: 0 - agudo; 1 - fracamente agudo/arredondado

7. Pubescência do clípeo: 0 - terço superior ou menos; 1 - metade superior ou maior

8. Cerdas no clípeo: 0 - predominantemente no primeiro terço; 1 - por todo o clípeo

9. Margem lateral do clípeo: 0 - sinuosa; 1 - reta

10. Contato clípeo-olho: 0 - clípeo toca o olho; 1 - clípeo afastado do olho

11. Pontuação na fronte e vértice: 0 - ausente ou espaçada e rasa; 1 - presente, densa e profunda 12. Espaço malar: 0 - menor ou igual ao quarto segmento da antena, 1 - maior que o quarto segmento da antena

13. Tamanho da Gena: 0 - mais estreito que o olho; 1 - igual ou mais largo que o olho

14. Distribuição da pubescência na Gena: 0 - do ápice até a metade, 1 - por toda a Gena

15. Carena occipital: 0 - presente; 1 - ausente

16. Carena pronotal: 0 - ausente ou fraca; 1 - presente e não produzida; 2 - presente e fortemente produzida, 3 - presente e aberrante

17. Sulco dorsal: 0 - presente; 1 - ausente

18. Sulco escrobal: 0 - evanescente/traço; 1 - larga; 2 - estreita; 3 - ausente

19. Forma do escutelo em vista lateral: 0 - angular; 1 - arredondado

20. Pontuações no escuto: 0 - ausente; 1 - espaçada e fina; 2 - densa e profunda

21. Visão lateral do metanoto: 0 - longo e curvado; 1 - curto e comprimido, 2 - vertical

22. Forma da valva do propódeo: 0 -arredondada; 1 - truncada

23. Concavidade propodeal: 0 - estreita; 1 - larga e rasa; 2 - larga e profunda; 3 - propódeo quase chato

24. Forma do segmento metassomal 1: 0 - alongado; 1 - conicamente séssil; 2 - conicamente peciolado

25. Tergo 1: 0 - igual ou mais longo que o tergo $2 ; 1$ - mais curto que o tergo 2

26. Tamanho do Tergo 2: 0 - mais longo do que largo; 1 - tão longo quanto largo; 2 - mais largo do que longo

27. Tamanho do prestígma: 0 - tão longo quanto largo; 1 - mais longo do que largo

28. Tamanho de $\mathrm{Cu} 1$ da asa posterior: 0 - tão comprido quando $\mathrm{Cu}-\mathrm{a} ; 2$ - mais curta que $\mathrm{Cu}-\mathrm{a}$

29. Posição da entrada do ninho: 0 - inferior; 1 - inferior

30. Favo: 0 - séssil; 1 - horizontal, suspensos pelo centro; 2- inclinado, suspensos pelos cantos

31. Sulco central no ninho: 0 - ausente; 1 - presente

32. Nervuras paralelas as linhas de construção: 0 - presente; 1 - ausente

33. Manchas de pulpas coloridas: 0 - presente; 1 - ausente

34. Angulo basal do paramero: 0 - obtuso; 1 - agudo

35. Angulo apical do paramero: 0 - largo; 1 - estreito

36. Processo ventral do edeago: 0 - curto e angular; 1 - estreito e reduzido

37. Forma do digito: 0 - arredondado; 1 - pontiagudo

38. Cerdas no espinho do parâmero: 0 - ausente; 1 - curta 2 - longa

A partir dos caracteres levantados foi gerado um cladograma (Figura 1) que demonstra a relação filogenética das espécies de Leipomeles e sua monofilia, corroborando a sinonímia feita por Carpenter (2004). Os caracteres observados como sinapomorfias que unem o grupo são: "Forma do primeiro segmento metassomal conicamente peciolado", diferente do grupo externo onde se encontram formas ou conicamente sésseis ou alongadas; "Presença de um sulco central na parede do ninho" e o "Ângulo apical do paramero estreito". O gênero Leipomeles também possui uma homoplasia com o gênero Chartergellus onde os ninhos de ambos possuem nervuras paralelas as linhas de construção. O clado formado por L. dorsata e L. spilogastra possui uma reversão quanto ao caráter do tamanho do tergo 1 , sendo "menor que o tergo 2" para os táxons de todo grupo que compartilham como característica a cerda curvada no terceiro palpomero labial, mas diferente apenas neste clado de L. dorsata e L. spilogastra, onde apresentam como estado "igual ou mais longo que o tergo 2" semelhante aos gêneros Angiopolybia e Agelaia. O clado formado por L. pusilla e L. albogrisea possuem como 
sinapomorfia, o tamanho da veia $\mathrm{Cu} 1$ da asa posterior sendo "tão comprido quando a veia $\mathrm{Cu}$ a", além disso o mesmo clado possui diversas homoplasias com outros gêneros, sendo estas, a forma do ápice do clípeo "fracamente agudo ou arredondado" compartilhada com Pseudopolybia, presença de pontuações na fronte e vértice "densa e profunda" e forma do metanoto em vista lateral "curto e comprimido", ambas características divididas com o clado formado por Chartergellus e Nectarinella, e o tamanho da gena "mais estreita que o olho" dividida com Angiopolybia. Foram identificados cinco espécimes machos da espécie Leipomeles dorsata e um espécime macho da espécie Leipomeles spilogastra, além de identificado um espécime que possivelmente é da espécie Leipomeles pusilla, único da coleção.

Tabela 1: Matriz de dados.

\begin{tabular}{|c|c|c|c|c|c|c|c|c|c|c|c|c|c|c|c|c|c|c|c|c|c|c|c|c|c|c|c|c|c|c|c|c|c|c|c|c|c|}
\hline & 01 & 2 & 3 & 4 & 56 & 7 & 8 & 9 & $\begin{array}{l}1 \\
\mathbf{0}\end{array}$ & $\begin{array}{l}1 \\
1\end{array}$ & $\begin{array}{l}1 \\
2\end{array}$ & $\begin{array}{l}1 \\
3\end{array}$ & $\begin{array}{l}1 \\
4\end{array}$ & $\begin{array}{l}1 \\
5\end{array}$ & $\begin{array}{l}1 \\
6\end{array}$ & $\begin{array}{l}1 \\
7\end{array}$ & $\begin{array}{l}1 \\
8\end{array}$ & $\begin{array}{l}1 \\
9\end{array}$ & $\begin{array}{l}2 \\
0\end{array}$ & $\begin{array}{l}2 \\
1\end{array}$ & $\begin{array}{l}2 \\
2\end{array}$ & $\begin{array}{l}2 \\
3\end{array}$ & $\begin{array}{l}2 \\
4\end{array}$ & $\begin{array}{l}2 \\
5\end{array}$ & $\begin{array}{l}2 \\
6\end{array}$ & $\begin{array}{l}2 \\
7\end{array}$ & $\begin{array}{l}2 \\
8\end{array}$ & $\begin{array}{l}2 \\
9\end{array}$ & $\begin{array}{l}3 \\
\mathbf{0}\end{array}$ & $\begin{array}{l}3 \\
1\end{array}$ & $\begin{array}{l}3 \\
2\end{array}$ & $\begin{array}{l}3 \\
3\end{array}$ & $\begin{array}{l}3 \\
4\end{array}$ & $\begin{array}{l}3 \\
5\end{array}$ & $\begin{array}{l}3 \\
6\end{array}$ & $\begin{array}{l}3 \\
7\end{array}$ & $\begin{array}{l}3 \\
8\end{array}$ \\
\hline $\begin{array}{l}\text { Agelaia } \\
\text { centralis }\end{array}$ & 00 & $\mathbf{0}$ & 0 & 0 & 00 & $\mathbf{0}$ & 0 & 0 & 0 & 0 & 1 & 0 & 0 & 1 & 0 & 0 & 0 & 0 & 0 & 0 & 0 & 0 & 0 & 2 & 0 & 1 & - & 1 & 0 & 1 & 0 & 1 & 0 & 0 & 1 & 0 & 0 \\
\hline $\begin{array}{l}\text { Angioploybia } \\
\text { pallens }\end{array}$ & 00 & 0 & 0 & 0 & 00 & 0 & 1 & 0 & 0 & 0 & 0 & 1 & 0 & 0 & 0 & 0 & 0 & 0 & 0 & 0 & 0 & 0 & 0 & 1 & 1 & 1 & 0 & 1 & 0 & 1 & 0 & 1 & 0 & 1 & 0 & 0 & 0 \\
\hline $\begin{array}{l}\text { Pseudopolybia } \\
\text { vespiceps }\end{array}$ & 10 & 0 & 1 & 0 & 01 & 0 & 0 & 1 & 0 & 1 & 1 & 0 & 1 & 0 & 0 & 1 & 0 & 0 & 0 & 1 & 1 & 1 & 1 & 1 & 1 & 1 & 0 & 1 & 0 & 1 & 0 & 0 & 0 & 0 & 1 & 0 & 2 \\
\hline $\begin{array}{l}\text { Parachartergus } \\
\text { fraternus }\end{array}$ & 10 & 1 & 0 & 0 & 10 & 1 & 1 & 1 & 0 & 0 & 1 & 1 & 0 & 3 & 1 & 3 & 0 & 1 & 0 & 1 & 2 & 1 & 1 & 2 & 1 & 1 & 0 & 2 & 0 & 1 & 1 & 0 & 0 & 1 & 0 & 1 & 1 \\
\hline $\begin{array}{l}\text { Chartergellus } \\
\text { communis }\end{array}$ & 11 & 1 & 1 & 0 & 10 & 1 & 0 & 1 & 1 & 0 & 1 & 1 & 1 & 2 & 1 & 2 & 0 & 1 & 1 & 1 & 3 & 1 & 1 & 0 & 0 & 1 & 0 & 2 & 0 & 0 & 1 & 0 & 0 & 1 & 1 & 1 & 0 \\
\hline $\begin{array}{l}\text { Nectarinella } \\
\text { xavantinensis }\end{array}$ & 11 & 1 & 1 & 0 & 10 & 1 & 1 & 1 & 1 & 0 & 1 & 1 & 1 & 2 & 1 & 1 & 1 & 2 & 2 & 1 & 3 & 1 & 1 & 2 & 0 & 1 & 0 & 0 & 0 & 1 & 1 & 1 & 0 & 0 & 1 & 1 & 0 \\
\hline $\begin{array}{l}\text { Leipomeles } \\
\text { dorsata }\end{array}$ & 11 & 1 & 1 & 1 & 00 & 0 & 1 & 0 & 0 & 0 & 1 & 0 & 0 & 0 & 1 & 0 & 0 & 0 & 0 & 0 & 0 & 2 & 0 & 1 & 0 & 1 & 1 & 2 & 1 & 0 & 1 & 1 & 1 & 1 & 0 & 1 & 0 \\
\hline $\begin{array}{l}\text { Leipomeles } \\
\text { spilogastra }\end{array}$ & 11 & 1 & 1 & 0,1 & 00 & 0 & 1 & 0 & 0 & 0 & 1 & 0 & 0 & 1 & 1 & 0 & 0 & 0 & 0 & 0 & 0 & 2 & 0 & 1 & 0 & 1 & $?$ & 2 & 1 & 0 & 1 & $?$ & $?$ & $?$ & $?$ & 1 & 0 \\
\hline $\begin{array}{l}\text { Leipomeles } \\
\text { albogrisea }\end{array}$ & 11 & 1 & 1 & 0 & $? 1$ & 1 & $?$ & 0 & 1 & 0 & 0 & $?$ & 0 & 1 & 1 & 0 & $?$ & 1 & 1 & 0 & $?$ & 2 & 1 & $?$ & 0 & 0 & 0 & 2 & 1 & 0 & 1 & 1 & 1 & 1 & 0 & $?$ & 0 \\
\hline $\begin{array}{l}\text { Leipomeles } \\
\text { pusilla }\end{array}$ & 11 & 1 & 1 & 0 & 01 & 1 & 1 & 0 & 1 & 0 & 0 & 0 & 0 & 1 & 1 & 0 & 0 & 1 & 1 & 0 & 1 & 2 & 1 & 0 & 0 & 0 & 0 & 2 & 1 & 0 & 1 & $?$ & $?$ & $?$ & $?$ & 1 & 0 \\
\hline
\end{tabular}

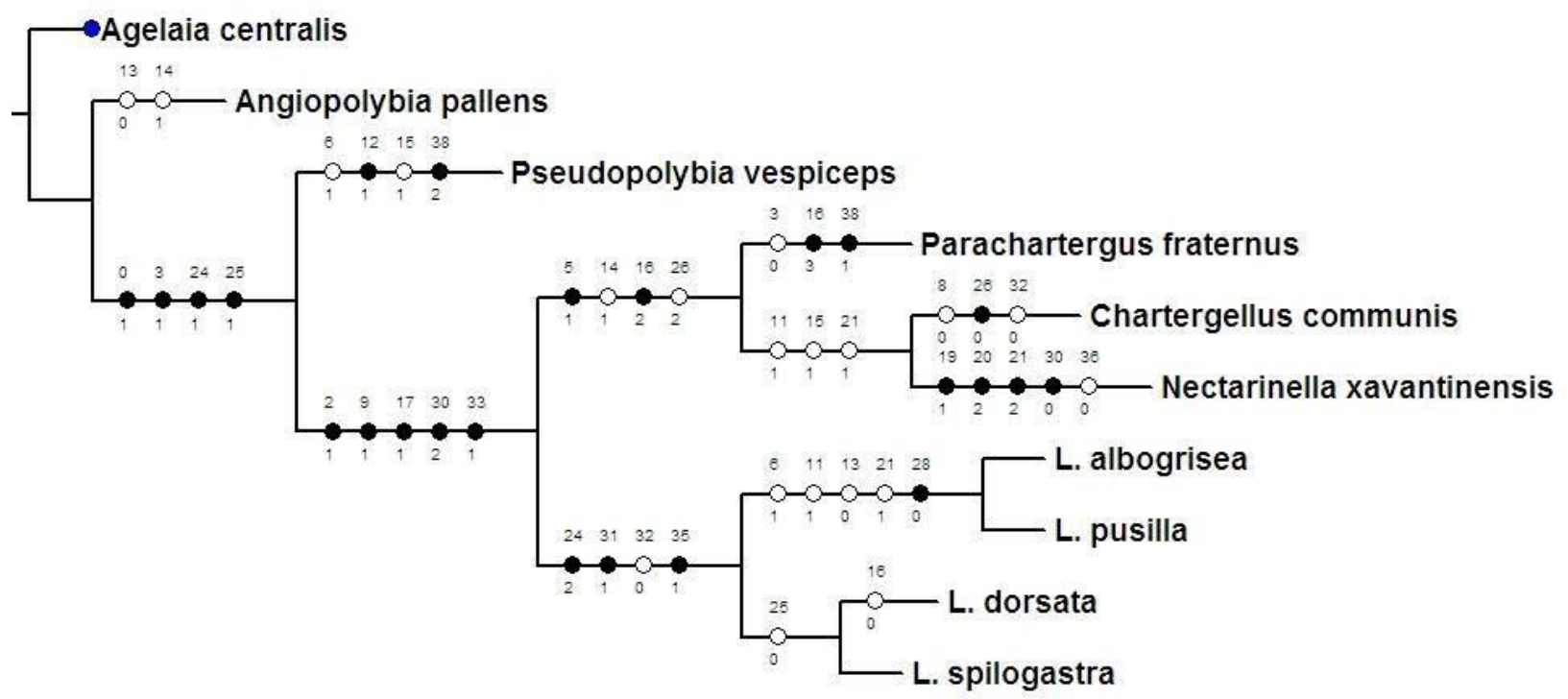

Figura 1: Relação filogenética entre táxons do grupo Leipomeles. 


\section{CONSIDERAÇÕES FINAIS}

Com a análise da matriz de caracteres pelo programa de parcimônia TNT foi corroborada a sinonímia do grupo estudado, demonstrando também a monofilia do gênero Leipomeles, ao todo são quatro sinapomorfias que unem o grupo, sendo elas a Forma do primeiro segmento metassomal, Sulco central no ninho, Veias paralelas as linhas de construção e Angulo apical do paramero. Embora as análises iniciais do projeto tenham obtido sucesso em comprovar a hipótese usando como base os caracteres disponíveis na literatura para Leipomeles albogrisea, ainda é necessário encontrar um espécime de L. albogrisea para dar mais consistência aos dados e testar se a monofilia se mantêm.

\section{REFERÊNCIAS}

Amorim, D. S. 1997. Elementos Básicos de Sistematica Filogenetica. Ribeirão Preto, São Paulo. Ed. Holos e Sociedade Brasileira de Entomologia.

Carpenter, J.M. 1991. Phylogenetic relationships and the origin of social behavior in the Vespidae. In K.G. Ross and R.W. Matthews (editors), The social biology of wasps: 7-32. Ithaca, NY: Cornell University Press.

Carpenter, J. M. 2004. Synonymy of the Genus Marimbonda Richards, 1978, with Leipomeles Möbius, 1856 (Hymenoptera: Vespidae; Polistinae), and a New Key to the Genera of Paper Wasps of the New World. American Museum of Natural History, New York. American Museum Novitates 3465.

Ducke, A. 1904. Sobre as Vespidas sociaes do Pará. Boletim do Museu Goeldi 4.

Goloboff, P., J. S. Farris and Nixon, K. 2008. TNT, a free program for phylogenetic analysis. Cladistics 24.

Henning, W. 1966. Phylogenetic Systematics. Urbana. Univ. Illinois Press.

Möbius, K.A. 1856. Die Nester der geselligen Wespen. Beschreibungen neuer Nester-und einigerneuen Wespen-arten des naturhistorischen Museums zu Hamburg nebst Betrachtungen über den Nesterbau im Allegemeinen. Abhandlungen des Naturwissenschaftlichen Vereins in Hamburg 3.

Nixon, K. C. 2002. Winclada, version 1.00000. Ithaca, NY: Publicado pelo autor.

Richards, O. W. 1978. The social wasps of the Americas (excluding the Vespinae). London, British Museum of Natural History.

Richards, O. W. 1978. The social wasps of the Americas (excluding the Vespinae). London, British Museum of Natural History.

Wenzel, J.W., and J.M. Carpenter. 1994. Comparing methods: Adaptive traits and tests of adaptation. In P. Eggleton and R.I. Vane-Wright (editors), Phylogenetics and Ecology: 79- 101. London: Academic Press. 